\title{
Gout, allopurinol intake and clinical outcomes in the hospitalized multimorbid elderly
}

\author{
Carlotta Franchi ${ }^{\text {a,* }}$, Francesco Salerno ${ }^{b}$, Alessio Conca ${ }^{b}$, Codjo D. Djade ${ }^{\text {a }}$, Mauro Tettamanti ${ }^{a}$, Luca Pasina ${ }^{\text {, }}$, \\ Salvatore Corrao ${ }^{c}$, Alessandra Marengoni ${ }^{\mathrm{d}}$, Maura Marcucci ${ }^{\mathrm{e}}$, \\ Pier Mannuccio Mannucci ${ }^{\mathrm{f}}$, Alessandro Nobili ${ }^{\mathrm{a}}$ \\ on behalf of the REPOSI Investigators ${ }^{1}$
}

\footnotetext{
a Department of Neuroscience, IRCCS - Istituto di Ricerche Farmacologiche "Mario Negri”, Milan, Italy

b Internal Medicine I, Policlinico IRCCS San Donato, University of Milan, Italy

c Dipartimento Biomedico di Medicina Interna e Specialistica, University of Palermo, Italy

${ }^{\mathrm{d}}$ Department of Clinical and Experimental Science, University of Brescia, Italy

e Department of Internal Medicine, IRCCS Ca' Granda Maggiore Policlinico Hospital Foundation, Milano, Italy

${ }^{f}$ Scientific Direction, IRCCS Maggiore Hospital Foundation, Milan, Italy
}

\section{A R T I C L E I N F O}

\section{Article history:}

Received 19 June 2014

Received in revised form 25 September 2014

Accepted 30 September 2014

Available online 22 October 2014

\section{Keywords:}

Gout

Allopurinol

Hyperuricemia

Comorbidities

Outcomes

Elderly

\begin{abstract}
A B S T R A C T
Background: Increased serum uric acid has been considered a cardiovascular risk factor but no study has assessed its relation with hospital mortality or length of stay. On the basis of data obtained from a prospective registry, the prevalence of gout/hyperuricemia and its association with these and other clinical parameters was evaluated in an Italian cohort of elderly patients acutely admitted to internal medicine or geriatric wards.

Methods: While the prevalence of gout was calculated by counting patients with this diagnosis hyperuricemia was inferred in patients taking allopurinol at hospital admission or discharge, on the assumption that this drug was only prescribed owing to the finding of high serum levels of uric acid. A series of clinical and demographic variables were evaluated for their association with gout/hyperuricemia.

Results: Of 1380 patients, 139 (10\%) had a diagnosis of gout or were prescribed allopurinol. They had more co-morbidities (7.0 vs 5.6; $\mathrm{P}<0.0001$ ) and consumed more drugs (6.8 vs 5.0; $\mathrm{P}<0.0001$ ). The CIRS (co-morbidity index) was worse in these patients (OR 1.28 95\% CI 1.15-1.41). Multivariable regression analysis showed that only renal and heart failures were independently associated with gout/allopurinol intake. Moreover, this combined event was associated with an increased risk of adverse events during hospitalization (OR 1.66, 95\% CI 1.16-2.36), but not with the risk of re-hospitalization, length of hospital stay or death.

Conclusions: Gout/allopurinol intake has a high prevalence in elderly patients acutely admitted to hospital and are associated with renal and cardiovascular diseases, an increased rate of adverse events and a high degree of drug consumption. In contrast, this finding did not affect the length of hospitalization nor hospital mortality.
\end{abstract}

(C) 2014 European Federation of Internal Medicine. Published by Elsevier B.V. All rights reserved.

\section{Introduction}

Gout is a clinical manifestation of hyperuricemia that leads to the deposition of urate crystals into joints, causing local reactions with typical signs and symptoms of inflammation. The clinical picture of gout may develop in subjects predisposed to produce high amounts of uric acid. More commonly, given that uric acid is eliminated through the kidney, patients with decreased glomerular filtration rate have

\footnotetext{
* Corresponding author at: Laboratory for Quality Assessment of Geriatric Therapies and Services, IRCCS - Istituto di Ricerche Farmacologiche "Mario Negri", Via La Masa 19, 20156 Milano, Italy. Tel.: + 3902 39014580; fax: + 390239001916.

E-mail address: carlotta.franchi@marionegri.it (C. Franchi).

1 REPOSI denotes Registry of Polytherapies SIMI (Società Italiana di Medicina Interna).
}

elevated serum concentrations. Gout prevalence has been increasing in recent years and is currently one of the most common causes of inflammatory arthritis [1]. This disease impacts on healthcare institutions in terms of health costs and utilization of healthcare services [2]. For instance, Robinson et al. reported an increasing trend in hospital admission due to gout from 1999 to 2009 [3]. In-hospital gout represents a significant patient and health care burden, with patients staying 6-7 days longer in hospital [4].

Gout is typically an age-associated disease, because uric acid deposition progressively increases over the years and gout flares are correlated with older age [5]. With this background, the aim of this study was to estimate the prevalence of gout and/or hyperuricemia prompting the prescription of allopurinol in an Italian population of patients aged 65 years or older acutely admitted to internal medicine or geriatric wards, during four different weeks representing the 
4 seasons over one year. Furthermore, secondary aims were to investigate whether or not gout/allopurinol intake in the elderly was associated with other demographic or clinical variables, in a particular length of hospital stay and mortality.

\section{Methods}

\subsection{Study setting and design}

This study was conducted in 66 hospital wards (representative of the Italian internal and geriatric medicine wards), participating during 2010 in the 'Registro Politerapie SIMI' (REPOSI). REPOSI is a collaborative and independent registry run by the Italian Society of Internal Medicine (SIMI), the Mario Negri Institute of Pharmacological Research and the IRCCS Ca' Granda Maggiore Policlinico Hospital Foundation in Milan. The detailed design of REPOSI is described elsewhere [6,7]. In brief, patients aged 65 years or more consecutively admitted to hospital during a period of four weeks, three months apart from each other, were enrolled in the registry. A standardized web-based case report form was filled in by the attending physicians, including socio-demographic factors, clinical parameters, diagnoses and medications prescribed both at hospital admission and discharge, adverse events (AEs) during hospitalization, co-morbidities according to the Cumulative Illness Rating Scale (CIRS) [8], performance in basic activities of daily living according to the Barthel Index scale [9], cognitive status according to the Short Blessed Test (SBT) [10] and presence of depression according to the Geriatric Depression Scale (GDS) [11]. All these data were collected and checked for consistency and accuracy, with possible contacts of the external contributors, by a central monitoring institution (the Mario Negri Institute for Pharmacological Research, Milan).

\subsection{Cohort composition}

All patients 65 years or older with a diagnosis of gout (International Classification of Disease 9th Edition (ICD-9): 274.xx) made at hospital admission or during hospital stay or in therapy with allopurinol (Anatomic Therapeutic Classification (ATC): M04AA01) were included in the study. Because serum levels of uric acid were not reported in the case report form, it was assumed that the prescription of allopurinol unrelated to the diagnosis of gout was triggered by the finding of high uric acid serum levels. Patients taking allopurinol for chemotherapy or chronic renal failure were excluded $(n=12)$. Co-morbidities were recorded according to the Cumulative Illness Rating Scale (CIRS). The CIRS co-morbidity index was computed by counting the number of items for which moderate to severe illness was reported (scores of 3, 4 or 5), while overall illness severity was represented by the mean of the 13 CIRS items [8]. The study was approved by the Ethical Committees of the IRCCS Cà Granda Maggiore Policlinico Hospital Foundation, Milan, and of all participating hospitals.

\subsection{Statistical analysis}

Univariable analyses were applied to compare patients with gout or treated with allopurinol versus those without these features, and data were described using means and standard deviations (SD) for numeric variables or number and percentages for categorical variables, compared applying univariate logistic regression and reported as odds ratios (OR) and 95\% confidence intervals (95\% CI). Multivariable logistic regression analyses assessed the association between gout/allopurinol intake and presence of other diseases. Selection of variables to be included in the multivariate model was based on statistical and clinical significance. The analysis was repeated using two different approaches for co-morbidity: first it was adjusted by sex, age, CIRS co-morbidity index and adverse events, then relevant concomitant diseases replaced the co-morbidity index. Further analyses were directed to assess whether or not gout/allopurinol intake were predictors of adverse events, mortality at discharge, increased length of hospital stay and readmission. All statistical calculations were performed with the software JMP Pro 10 (SAS Institute Inc.).

\section{Results}

Of 1380 enrolled patients, 1368 (99\%) were included in the study: $139(10 \%)$ had a diagnosis of gout or were taking allopurinol (exposed group), whereas the remaining 1229 represented the non-exposed group (Table 1). Table 1 also shows the socio-demographic and clinical characteristics of the exposed and non-exposed patients and factors associated with gout diagnosis or allopurinol intake. Even though 65 years was the limit for admission to the study, the average patient age was much higher, with no statistically significant difference between the two groups even though the exposed patients tended to be older.

By univariable analysis, the exposed group showed the following statistically significant differences in comparison with the nonexposed group. Patients with gout/allopurinol intake were more often males ( $61 \%$ vs $48 \%$; $\mathrm{P}=0.003$ ) and their body mass index (BMI) was higher than that of the remaining patients (27.7 vs $25.8 \% ; \mathrm{P}=0.0004)$. Patients with gout/allopurinol intake was also characterized by a higher number of concomitant diagnoses at hospital admission (mean: 6.8 vs 5.6 ; $\mathrm{P}<0.0001$ ) and consumed more drugs other than allopurinol ( 6.8 vs $5.0 ; \mathrm{P}<0.0001)$ : more sulfonamides (73\%), proton pump inhibitors (52\%), statins (32\%), vitamin $\mathrm{K}$ antagonists (27\%), nitrates (26\%), beta blocking agents (25\%) and angiotensin-II antagonists (23\%). At admission the CIRS co-morbidity index (3.6 vs 2.8; $\mathrm{P}<0.001$ ) and the severity index (1.8 vs $1.6, \mathrm{P}<0.001$ ) were worse in patients with gout/allopurinol intake. The diagnoses more frequently associated to gout/allopurinol intake were hypertension ( $85 \%$ vs $76 \%, \mathrm{P}=0.011$ ), chronic renal failure ( $45 \%$ vs $14 \%, \mathrm{P}<0.0001$ ), diabetes ( $37 \%$ vs $26 \%, \mathrm{P}=0.005$ ), heart failure ( $37 \%$ vs $14 \%, \mathrm{P}<0.0001)$ and coronary artery disease (30\% vs $21 \%, \mathrm{P}=0.025$ ).

On the other hand, there was no difference between the two groups for the rates of atrial fibrillation, COPD, cerebrovascular disease and malignancy. Moreover, there was no significant difference pertaining to smoking and alcohol intake, Barthel Index, SBT and GDS at admission, nor for duration of hospital stay and mortality. After adjustment for variables statistically significant at univariable analysis, the multivariable regression analyses (Table 2 ) restricted the number of significantly different variables only to chronic renal failure (OR 3.56, 95\% CI 2.39-5.31) and heart failure (OR 2.63, 95\% CI 1.75-3.92).

Table 2 also shows that having a diagnosis of gout or taking allopurinol was also associated to an increased risk to have adverse events (AEs) during hospitalization (OR 1.66, 95\% IC 1.16-2.36), but not with the risk of re-hospitalization, length of hospital stay or in-hospital mortality. The most frequent AEs were urinary tract infections (10.1\%), hypokalemia (3.6\%), anemia (3.6\%), pneumonia (3.6\%), heart failure (3.6\%), respiratory tract infections (2.8\%), atrial fibrillation (2.1\%), fever (1.4\%), renal failure (1.4\%) and bacteremia (1.4\%).

\section{Discussion}

This study shows that gout and/or allopurinol intake presumably due to hyperuricemia is very frequent diagnoses in elderly patients admitted to internal medicine or geriatric wards, because they were reported in about $10 \%$ of people acutely admitted to the hospital because of different causes and diagnoses. This prevalence is high, especially considering that the prevalence of gout is $0.46 \%$ in the Italian general population [12], similar to that of other Mediterranean countries [13]. It must be considered that prevalence of gout and hyperuricemia are rapidly growing and that the population analyzed was at high risk, being old and hospitalized. Only cardiac and renal failures 
Table 1

Sociodemographic and clinical characteristics of patients with or without gout/allopurinol intake (univariable analysis).

\begin{tabular}{|c|c|c|c|c|}
\hline \multicolumn{5}{|l|}{ Socio-demographic and clinical characteristics } \\
\hline \multirow[t]{2}{*}{ Variables } & \multicolumn{4}{|c|}{ Allopurinol intake or gout } \\
\hline & Yes $(\mathrm{N}=139)$ & No $(\mathrm{N}=1229)$ & Odds ratio $(95 \% \mathrm{CI})$ & P value \\
\hline Age, mean (SD) & $80.0(7.1)$ & $78.8(7.3)$ & $1.02(1.00-1.04)$ & 0.058 \\
\hline \multicolumn{5}{|l|}{ Risk factors, $\mathrm{N}(\%)$} \\
\hline Smoker & $14(10.0)$ & $109(8.8)$ & $1.15(0.63-2.06)$ & 0.65 \\
\hline Heavy alcohol drinker & $70(50.3)$ & $510(41.6)$ & $1.42(1.00-2.01)$ & 0.051 \\
\hline Number of patients at admission with 5 or more diagnoses, mean (SD) & $91(65.4)$ & $588(47.9)$ & $2.06(1.42-2.97)$ & $<.0001$ \\
\hline Number of patient at admission taking 5 or more drugs, $\mathrm{N}(\%)$ & $113(81.2)$ & $675(54.9)$ & $3.57(2.33-5.65)$ & $<.0001$ \\
\hline Severity index at admission, mean (SD) & $1.8(0.3)$ & $1.6(0.3)$ & $5.13(3.02-8.71)$ & $<.0001$ \\
\hline CIRS comorbidity index at admission, mean (SD) & $3.6(1.6)$ & $2.8(1.7)$ & $1.28(1.15-1.41)$ & $<.0001$ \\
\hline Barthel at admission, mean (SD) & $77.6(32.4)$ & $77.4(30.3)$ & $1.00(1.00-1.00)$ & 0.101 \\
\hline Short blessed test, mean (SD) & $10.9(8.8)$ & $9.7(8.0)$ & $1.02(0.99-1.04)$ & 0.141 \\
\hline Geriatric depression scale, mean (SD) & $1.5(1.2)$ & $1.4(1.2)$ & $1.13(0.98-1.30)$ & 0.107 \\
\hline Duration of hospital stay, mean (SD) & $10.9(8.9)$ & $10.7(8.1)$ & $1.00(0.98-1.02)$ & 0.787 \\
\hline Adverse clinical events during hospitalization, $\mathrm{N}(\%)$ & $63(45.3)$ & $410(33.3)$ & $1.65(1.16-2.36)$ & 0.006 \\
\hline Hospital mortality, $\mathrm{N}(\%)$ & $8(5.8)$ & $40(3.3)$ & $1.81(0.83-3.95)$ & 0.160 \\
\hline
\end{tabular}

were significantly associated with gout/allopurinol intake. These associations can be attributed to the well established risk to develop cardiovascular complication in patients with urate overproduction/ decreased elimination $[14,15]$. Another hypothesis is that many patients with gout or hyperuricemia share with those with cardiovascular or renal diseases some frequent risk factors such as overweight, hypertension, coronary artery disease and diabetes [16,17].

Another finding of this study was that patients with gout/allopurinol intake experienced during hospitalization a higher number of AEs that were often severe, including bacterial infections, heart and renal failure and arrhythmias. Accordingly, particular care and attention should be devoted when elderly patients with gout or allopurinol therapy are hospitalized pertaining to the functions of the cardiovascular system and the kidney. Prevention of these complications might be obtained by avoiding excessive diuretic medications in patients with increased serum creatinine according to the definition of acute kidney injury (an increase of $50 \%$ above the basal value or of $0.3 \mathrm{mg} / \mathrm{dl}$ within $48 \mathrm{~h}$ ) and, on the other hand, by reducing or stopping fluid infusion in those with early signs of impending cardiac failure. Another very important

Table 2

Association of gout and/or allopurinol intake with the incidence of adverse events during hospitalization (univariable and multivariable analysis).

\begin{tabular}{|c|c|c|}
\hline Variables & OR $(95 \% \mathrm{CI})$ & P value \\
\hline \multicolumn{3}{|l|}{ 1. Univariable analysis } \\
\hline Patients with gout/allopurinol intake & $1.66(1.16-2.36)$ & 0.006 \\
\hline \multicolumn{3}{|c|}{ 2. Multivariable analysis adjusted for age, sex, AEs and CIRS comorbidity index. } \\
\hline Patients with gout/allopurinol intake & $1.52(1.06-2.19)$ & 0.024 \\
\hline Sex (female) & $0.93(0.74-1.18)$ & 0.557 \\
\hline \multicolumn{3}{|l|}{ Age groups } \\
\hline $65-75$ & 1.0 & 0.0005 \\
\hline $75-84$ & $1.29(0.99-1.69)$ & \\
\hline$>=85$ & $1.89(1.37-2.60)$ & \\
\hline CIRS comorbidity index & $1.07(1.00-1.15)$ & 0.036 \\
\hline \multicolumn{3}{|c|}{ 3. Multivariable analysis adjusted for age, sex and associated diseases. } \\
\hline Patients with gout/allopurinol intake & $1.48(1.02-2.16)$ & 0.041 \\
\hline Sex (female) & $0.92(0.73-1.16)$ & 0.493 \\
\hline \multicolumn{3}{|l|}{ Age groups } \\
\hline $65-75$ & 1 & 0.0004 \\
\hline $75-84$ & $1.30(1.00-1.71)$ & \\
\hline$>=85$ & $1.91(1.38-2.64)$ & \\
\hline Hypertension & $0.89(0.69-1.16)$ & 0.380 \\
\hline Chronic renal insufficiency & $1.27(0.93-1.73)$ & 0.138 \\
\hline Diabetes mellitus & $0.89(0.68-1.16)$ & 0.390 \\
\hline Heart failure & $1.13(0.83-1.53)$ & 0.431 \\
\hline Ischemic heart disease & $0.94(0.71-1.24)$ & 0.659 \\
\hline
\end{tabular}

recommendation is to implement any possible effort to reduce the burden of infectious agents in the environment.

Our study did not show a relationship between gout/allopurinol intake and an increased risk of re-hospitalization, length of hospital stay or in-hospital mortality. In contrast, a previous study [4] showed that hospitalized patients with acute gout stayed 6 days longer than controls, perhaps because gout was a complicating factor for hospitalization owing to other reasons. Moreover, Robinson et al. showed that patients with hospital admissions secondarily complicated by gout had a poorer survival compared with those admitted primarily for gout [3].

In general, the in-hospital management of elderly patients should take into account the frequent association with co-morbidities. The BMI of our cohort was indeed significantly higher in these patients, consistently with knowledge that overweight or obesity is a well established risk factor for abnormal glucose and lipid metabolisms. Unfortunately, the data collected in the REPOSI registry did not include also the serum levels of glucose, cholesterol and tryglicerides. However, patients with gout/allopurinol intake often took medications such as statins, which are prescribed in order to decrease serum lipid concentrations. Furthermore, at univariate analysis, gout/allopurinol intake was associated with the diagnosis of diabetes, suggesting once more that similar pathogenic mechanisms exist for gout, hyperuricemia, diabetes and dyslipidemia. Indeed, studies in elderly people with pre-diabetes suggested that high uric acid levels raise a personal chance to become diabetic [18].

The present study has also shown that patients with gout and/or allopurinol intake took a greater number of drugs to treat the frequent multiple concomitant diseases. This prescription behavior raises the issue of the usefulness of basing drug prescription solely on the simple sum of different diagnoses, because in the elderly the effects of many drugs may be magnified or reduced due to pharmacodynamic changes and/or important interactions between the many prescribed medications. Accordingly, an individually tailored therapy should take into account the risk of interactions with concurrent medications. In particular, drugs with an excretion pathway common to that of uric acid should be prescribed with caution, because in these instances side effects could be early and severe.

The present study has limitations. The main weakness was that the diagnosis of high serum concentrations of uric acid was not based on the direct dosage of this moiety in patient serum, but mainly inferred from the finding of allopurinol intake. This assumption was based on the belief that it is unlikely that a drug not free of adverse events such as allopurinol is prescribed in the presence of normal serum levels of uric acid, even though we are also cognizant that not all patients with hyperuricemia benefit from allopurinol intake. By contrast, there are also some strength that are mainly related to the prospective data 
collection and the participation of more than 70 wards of general hospitals, making the study representative of a wide area in Southern Europe.

\section{Learning points}

- Gout and/or hyperuricemia is frequent in elderly patients admitted to internal medicine wards.

- Only cardiac and renal failures were significantly associated with gout/allopurinol intake.

- Gout or taking allopurinol was associated to an increased risk of hospital adverse events.

- Gout or allopurinol intake should prompt to look for cardiovascular or renal diseases, drug interactions and clinical risks.

\section{Disclosure statement}

All authors have no conflicts of interests to disclose

\section{Appendix A. Investigators and co-authors of the REPOSI (REgistro POliterapie SIMI, Società Italiana di Medicina Interna) Study Group are as follows:}

Steering Committee: Pier Mannuccio Mannucci (Chair, Fondazione IRCCS Cà Granda Ospedale Maggiore Policlinico, Milano), Alessandro Nobili (co-chair, IRCCS-Istituto di Ricerche Farmacologiche "Mario Negri", Milano), Mauro Tettamanti, Luca Pasina, Carlotta Franchi (IRCCS-Istituto di Ricerche Farmacologiche "Mario Negri", Milano), Francesco Salerno (IRCCS Policlinico San Donato Milanese, Milano), Salvatore Corrao (ARNAS Civico, Di Cristina, Benfratelli, DiBiMIS, Università di Palermo, Palermo), Alessandra Marengoni (Spedali Civili di Brescia, Brescia), Maura Marcucci (Dipartimento di Medicina Interna, Fondazione IRCCS Cà Granda Ospedale Maggiore Policlinico, Milano).

Clincal data monitoring and revision: Eleonora Sparacio, Stefania Alborghetti, Rosa Di Costanzo (IRCCS-Istituto di Ricerche Farmacologiche "Mario Negri", Milano).

Database Management and Statistics: Mauro Tettamanti, Codjo Djignefa Djade (IRCCS-Istituto di Ricerche Farmacologiche "Mario Negri", Milano).

\section{Investigators:}

Domenico Prisco, Elena Silvestri, Caterina Cenci, Tommaso Barnini (Azienda Ospedaliero Universitaria Careggi Firenze, SOD Patologia Medica); Giuseppe Delitala, Stefano Carta, Sebastiana Atzori (Azienda Mista Ospedaliera Universitaria, Sassari, Clinica Medica); Gianfranco Guarnieri, Michela Zanetti, Annalisa Spalluti (Azienda Ospedaliera Universitaria Ospedali Riuniti di Trieste, Trieste, Clinica Medica Generale e Terapia Medica); Maria Grazia Serra, Maria Antonietta Bleve (Azienda Ospedaliera "Cardinale Panico" di Tricase, Lecce, Unità Operativa Complessa Medicina); Massimo Vanoli, Giulia Grignani, Gianluca Casella (Azienda Ospedaliera della Provincia di Lecco, Ospedale di Merate, Lecco, Medicina Interna); Laura Gasbarrone (Azienda Ospedaliera Ospedale San Camillo Forlanini, Roma, Medicina Interna 1); Giorgio Maniscalco, Massimo Gunelli, Daniela Tirotta (Azienda Ospedaliera Ospedale San Salvatore, Pesaro, Soc Medicina Interna); Antonio Brucato, Silvia Ghidoni, Paola Di Corato (Azienda Ospedaliera Papa Giovanni XXIII, Bergamo, Medicina 1); Mauro Bernardi, Silvia Li Bassi, Luca Santi (Azienda Ospedaliera Policlinico Sant'Orsola-Malpighi, Bologna, Semeiotica Medica Bernardi); Giancarlo Agnelli, Alfonso Iorio, Maura Marcucci, Emanuela Marchesini (Azienda Ospedaliera Santa Maria della Misericordia, Perugia, Medicina Interna e Cardiovascolare); Elmo Mannarino, Graziana Lupattelli, Pamela Rondelli, Francesco Paciullo (Azienda Ospedaliera Santa Maria della Misericordia, Perugia, Medicina Interna, Angiologia, Malattie da Arteriosclerosi); Fabrizio Fabris, Michela Carlon, Francesca
Turatto (Azienda Ospedaliera Università di Padova, Padova, Clinica Medica I); Maria Cristina Baroni, Marianna Zardo (Azienda Ospedaliera Università di Parma, Parma, Clinica e Terapia Medica); Roberto Manfredini, Christian Molino, Marco Pala, Fabio Fabbian (Azienda Ospedaliera - Universitaria Sant'Anna, Ferrara, Unità Operativa Clinica Medica); Ranuccio Nuti, Roberto Valenti, Martina Ruvio, Silvia Cappelli (Azienda Ospedaliera Università Senese, Siena, Medicina Interna I); Giuseppe Paolisso, Maria Rosaria Rizzo, Maria Teresa Laieta (Azienda Ospedaliera Universitaria della Seconda Università degli Studi di Napoli, Napoli, VI Divisione di Medicina Interna e Malattie Nutrizionali dell'Invecchiamento); Teresa Salvatore, Ferdinando Carlo Sasso (Azienda Ospedaliera Universitaria della Seconda Università degli Studi di Napoli, Napoli, Medicina Interna e Malattie Epato-Bilio Metaboliche Avanzate); Riccardo Utili, Emanuele Durante Mangoni, Daniela Pinto (Azienda Ospedaliera Universitaria della Seconda Università degli Studi di Napoli, Napoli, Medicina Infettivologica e dei trapianti); Oliviero Olivieri, Anna Maria Stanzial (Azienda Ospedaliera Universitaria Integrata di Verona, Verona, Unità Operativa di Medicina Interna B); Renato Fellin, Stefano Volpato, Sioulis Fotini (Azienda Ospedaliera Universitaria Ospedale Sant'Anna, Ferrara, Unità Operativa di Medicina Interna Gerontologia e Geriatria); Mario Barbagallo, Ligia Dominguez, Lidia Plances, Daniela D'Angelo (Azienda Ospedaliera Universitaria Policlinico Giaccone Policlinico di Palermo, Palermo, Unità Operativa di Geriatria e Lungodegenza); Giovanbattista Rini, Pasquale Mansueto, Ilenia Pepe (Azienda Ospedaliera Universitaria Policlinico P. Giaccone di Palermo, Palermo, Medicina Interna e Malattie Metaboliche); Giuseppe Licata, Luigi Calvo, Maria Valenti (Azienda Ospedaliera Universitaria Policlinico P. Giaccone di Palermo, Palermo, Medicina Interna e Cardioangiologia); Claudio Borghi, Enrico Strocchi, Elisa Rebecca Rinaldi (Azienda Ospedaliera Universitaria Policlinico S. Orsola-Malpighi, Bologna, Unità Operativa di Medicina Interna Borghi); Marco Zoli, Elisa Fabbri, Donatella Magalotti (Azienda Ospedaliera Universitaria Policlinico S. Orsola-Malpighi, Bologna, Unità Operativa di Medicina Interna Zoli); Alberto Auteri, Anna Laura Pasqui, Luca Puccetti (Azienda Ospedaliera Universitaria Senese, Siena, Medicina 3); Franco Laghi Pasini, Pier Leopoldo Capecchi, Maurizio Bicchi (Azienda Ospedaliera Universitaria Senese, Siena, Unità Operativa Complessa Medicina 2); Carlo Sabbà, Francesco Saverio Vella, Alessandro Marseglia, Chiara Valentina Luglio (Azienda Ospedaliero-Universitaria Consorziale Policlinico di Bari, Bari, Medicina Interna Universitaria C. Frugoni); Giuseppe Palasciano, Maria Ester Modeo, Annamaria Aquilino, Pallante Raffaele (Azienda Ospedaliero-Universitaria Consorziale Policlinico di Bari, Bari, Medicina Interna Ospedale "Pende-Ferrannini"); Stefania Pugliese, Caterina Capobianco (Azienda Ospedaliero-Universitaria Consorziale Policlinico di Bari, Bari, Clinica Medica I Augusto Murri); Alfredo Postiglione, Maria Rosaria Barbella, Francesco De Stefano (Azienda Ospedaliera Universitaria Policlinico Federico II di Napoli, Medicina Geriatrica Dipartimento di Clinica Medica); Luigi Fenoglio, Chiara Brignone, Christian Bracco, Alessia Giraudo (Azienda Sanitaria Ospedaliera Santa Croce e Carle di Cuneo, Cuneo, S. C. Medicina Interna); Giuseppe Musca, Olga Cuccurullo (Azienda Sanitaria Provinciale di Cosenza Presidio Ospedaliero di Cetraro, Cosenza, Unità Operativa Complessa Medicina Interna); Luigi Cricco, Alessandra Fiorentini ( $\mathrm{COB}$ Stabilimento Montefiascone, Viterbo, Unità Operativa Complessa di Geriatria e Medicina); Maria Domenica Cappellini, Giovanna Fabio, Sonia Seghezzi, Margherita Migone De Amicis (Fondazione IRCCS Cà Granda Ospedale Maggiore Policlinico, Milano, Unità Operativa Medicina Interna IA); Silvia Fargion, Paola Bonara, Mara Bulgheroni, Rosa Lombardi (Fondazione IRCCS Cà Granda Ospedale Maggiore Policlinico, Milano, Medicina Interna 1B); Fabio Magrini, Ferdinando Massari, Tatiana Tonella (Fondazione IRCCS Cà Granda Ospedale Maggiore Policlinico, Milano, Unità Operativa Medicina Cardiovascolare); Flora Peyvandi, Alberto Tedeschi, Raffaella Rossio (Fondazione IRCCS Cà Granda Ospedale Maggiore Policlinico, Milano, Medicina Interna 2); Guido Moreo, Barbara Ferrari, Luisa Roncari (Fondazione IRCCS Cà Granda Ospedale Maggiore Policlinico, Milano, Medicina Interna 3); Valter 
Monzani, Valeria Savojardo, Christian Folli, Maria Magnini (Fondazione IRCCS Cà Granda Ospedale Maggiore Policlinico, Milano, Medicina d'Urgenza); Daniela Mari, Paolo Dionigi Rossi, Sarah Damanti, Silvia Prolo (Fondazione IRCCS Cà Granda Ospedale Maggiore Policlinico, Milano, Geriatria); Maria Sole Lilleri (Fondazione IRCCS Cà Granda Ospedale Maggiore Policlinico, Milano, Medicina Generale ad Indirizzo Geriatrico); Luigi Cricco, Alessandra Fiorentini (COB Viterbo, Stabilimento Montefiascone, Viterbo, UOC Geriatria e Medicina); Giuliana Micale (IRCCS Istituto Auxologico Italiano, Milano, Medicina Generale ad indirizzo Geriatrico); Mauro Podda, Carlo Selmi, Francesca Meda (IRCCS Istituto Clinico Humanitas, Milano, Clinica Medica); Francesco Salerno, Silvia Accordino, Alessio Conca, Valentina Monti (IRCCS Policlinico San Donato e Università di Milano, San Donato Milanese, Medicina Interna); Gino Roberto Corazza, Emanuela Miceli, Marco Vincenzo Lenti, Donatella Padula (IRCCS Policlinico San Matteo di Pavia, Pavia, Clinica Medica I, Reparto 11); Carlo L. Balduini, Giampiera Bertolino, Stella Provini, Federica Quaglia (IRCCS Policlinico San Matteo di Pavia, Pavia, Clinica Medica III); Giovanni Murialdo, Marta Bovio (IRCS Azienda Ospedaliera Universitaria San Martino-IST di Genova, Genova, Clinica di Medicina Interna 2); Franco Dallegri, Luciano Ottonello, Alessandra Quercioli, Alessandra Barreca (Università di Genova, Genova, Medicina Interna 1); Maria Beatrice Secchi, Davide Ghelfi (Ospedale Bassini di Cinisello Balsamo, Milano, Divisione Medicina); Wu Sheng Chin, Laura Carassale, Silvia Caporotundo (Ospedale Bassini, Cinisello Balsamo, Milano, Unità Operativa di Geriatria); Luigi Anastasio, Lucia Sofia, Maria Carbone (Ospedale Civile Jazzolino di Vibo Valentia, Vibo Valentia, Medicina interna); Giancarlo Traisci, Lucrezia De Feudis, Silvia Di Carlo (Ospedale Civile Santo Spirito di Pescara, Pescara, Medicina Interna 2); Giovanni Davì, Maria Teresa Guagnano, Simona Sestili (Ospedale Clinicizzato SS. Annunziata, Chieti, Clinica Medica); Elisabetta Bergami, Emanuela Rizzioli (Ospedale del Delta, Lagosanto, Ferrara, Medicina Interna); Carlo Cagnoni, Luca Bertone, Antonio Manucra (Ospedale di Bobbio, Piacenza, Unità Operativa Medicina e Primo Soccorso); Alberto Buratti, Tiziana Tognin, Nicola Lucio Liberato (Azienda Ospedaliera della Provincia di Pavia, Ospedale di Casorate Primo, Pavia, Medicina Interna); Giordano Bernasconi, Barbara Nardo (Ospedale di Circolo di Busto Arsizio, Varese, Medicina I); Giovanni Battista Bianchi, Sabrina Giaquinto Ospedale "SS Gerosa e Capitanio" di Lovere, Bergamo, Unità Operativa Complessa di Medicina Generale, Azienda Ospedaliera “Bolognini” di Seriate, Bergamo; Giampiero Benetti, Michela Quagliolo, Giuseppe Riccardo Centenaro (Ospedale di Melegnano, Vizzolo Predabissi, Melegnano, Medicina 1). Francesco Purrello, Antonino Di Pino, Salvatore Piro (Ospedale Garibaldi Nesima, Catania, Unità Operativa Complessa di Medicina Interna); Gerardo Mancuso, Daniela Calipari, Mosè Bartone, Francesco Gullo (Ospedale Giovanni Paolo II Lamezia Terme, Catanzaro, Unità Operativa Complessa Medicina Interna); Michele Cortellaro, Marina Magenta, Francesca Perego; Maria Rachele Meroni (Ospedale Luigi Sacco, Milano, Medicina $3^{\circ}$ ); Marco Cicardi, Antonio Gidaro Marina Magenta (Ospedale Luigi Sacco, Milano, Medicina II); Andrea Sacco, Antonio Bonelli, Gaetano Dentamaro (Ospedale Madonna delle Grazie, Matera, Medicina); Renzo Rozzini, Lina Falanga, Alessandro Giordano (Ospedale Poliambulanza, Brescia, Medicina Interna e Geriatria); Paolo Cavallo Perin, Bartolomeo Lorenzati, Gabriella Gruden, Graziella Bruno (Dipartimento di Scienze Mediche, Università di Torino, Città della Scienza e della Salute, Torino, Medicina 3); Giuseppe Montrucchio, Elisabetta Greco, Pietro Tizzani (Dipartimento di Scienze Mediche, Università di Torino, Città della Scienza e della Salute, Torino, Medicina Interna 5); Giacomo Fera, Maria Loreta Di Luca, Donatella Renna (Ospedale San Giacomo di Monopoli, Bari, Unità Operativa Medicina Interna); Antonio Perciccante, Alessia Coralli (Ospedale San Giovanni-Decollato-Andisilla, Civita Castellana Medicina); Rodolfo Tassara, Deborah Melis, Lara Rebella (Ospedale San Paolo, Savona, Medicina I); Giorgio Menardo, Stefania Bottone, Elsa Sferrazzo (Ospedale San Paolo, Savona, Medicina Interna e Gastroenterologia); Claudio Ferri, Rinaldo Striuli, Rosa Scipioni (Ospedale San Salvatore, L'Aquila, Medicina Interna Universitaria); Raffaella Salmi, Piergiorgio Gaudenzi, Susanna Gamberini, Franco Ricci (Azienda Ospedaliera-Universitaria
S. Anna, Ferrara, Unità Operativa di Medicina Ospedaliera II); Cosimo Morabito, Roberto Fava (Ospedale Scillesi d'America, Scilla Medicina); Andrea Semplicini, Lucia Gottardo (Ospedale SS. Giovanni e Paolo, Venezia, Medicina Interna 1); Giuseppe Delitala, Stefano Carta, Sebastiana Atzori (Ospedale Universitario Policlinico di Sassari, Sassari, Clinica Medica); Gianluigi Vendemiale, Gaetano Serviddio, Roberta Forlano (Ospedali Riuniti di Foggia, Foggia, Medicina Interna Universitaria); Luigi Bolondi, Leonardo Rasciti, Ilaria Serio (Policlinico Sant'Orsola-Malpighi, Bologna, Unità Operativa Complessa Medicina Interna); Cesare Masala, Antonio Mammarella, Valeria Raparelli (Policlinico Umberto I, Roma, Medicina Interna D); Filippo Rossi Fanelli, Massimo Delfino, Antonio Amoroso (Policlinico Umberto I, Roma, Medicina Interna H); Francesco Violi, Stefania Basili, Ludovica Perri (Policlinico Umberto I, Roma, Prima Clinica Medica); Pietro Serra, Vincenzo Fontana, Marco Falcone (Policlinico Umberto I, Roma, Terza Clinica Medica); Raffaele Landolfi, Antonio Grieco, Antonella Gallo (Policlinico Universitario A. Gemelli, Roma, Clinica Medica); Giuseppe Zuccalà, Francesco Franceschi, Guido De Marco, Cordischi Chiara, Sabbatini Marta (Policlinico Universitario A. Gemelli, Roma, Roma, Unità Operativa Complessa Medicina d'Urgenza e Pronto Soccorso); Martino Bellusci, Donatella Setti, Filippo Pedrazzoli (Presidio Ospedaliero Alto Garda e Ledro, Ospedale di Arco, Trento, Unità Operativa di Medicina Interna Urgenza/Emergenza); Giuseppe Romanelli, Caterina Pirali, Claudia Amolini (Spedali Civili di Brescia, Brescia, Geriatria); Enrico Agabiti Rosei, Damiano Rizzoni, Luana Castoldi (Spedali Civili di Brescia, Brescia, Seconda Medicina); Antonio Picardi, Umberto Vespasiani Gentilucci, Chiara Mazzarelli, Paolo Gallo (Università Campus Bio-Medico, Roma, Medicina Clinica-Epatologia); Luigina Guasti, Luana Castiglioni, Andrea Maresca, Alessandro Squizzato, Sara Contini, Marta Molaro (Università degli Studi dell'Insubria, Ospedale di Circolo e Fondazione Macchi, Varese, Medicina Interna I); Giorgio Annoni, Maurizio Corsi, Sara Zazzetta (Università degli studi di Milano-Bicocca Ospedale S. Gerardo, Monza, Unità Operativa di Geriatria); Marco Bertolotti, Chiara Mussi, Roberto Scotto, Maria Alice Ferri, Francesca Veltri (Università di Modena e Reggio Emilia, AUSL di Modena, Modena, Nuovo Ospedale Civile, Unità Operativa di Geriatria); Franco Arturi, Elena Succurro, Giorgio Sesti, Umberto Gualtieri (Università degli Studi Magna Grecia, Policlinico Mater Domini, Catanzaro, Unità Operativa Complessa di Medicina Interna); Francesco Perticone, Angela Sciacqua, Michele Quero, Chiara Bagnato (Università Magna Grecia Policlinico Mater Domini, Catanzaro, Unità Operativa Malattie Cardiovascolari Geriatriche); Paola Loria, Maria Angela Becchi, Gianfranco Martucci, Alessandra Fantuzzi, Mauro Maurantonio (Università di Modena e Reggio Emilia, Medicina MetabolicaNOCSAE, Baggiovara, Modena); Roberto Corinaldesi, Roberto De Giorgio, Mauro Serra, Valentina Grasso, Eugenio Ruggeri, Lorenzo Mauro Carozza, Fabio Pignatti (Dipartimento di Scienze Mediche e Chirurgiche, Unità Operativa di Medicina Interna, Università degli Studi di Bologna/Azienda Ospedaliero-Universitaria S. Orsola-Malpighi, Bologna).

\section{References}

[1] Richette P, Bardin T. Gout. Lancet 2010;375:318-28.

[2] Trieste L, Palla I, Fusco F, Tani C, Baldini C, Mosca M, et al. The economic impact of gout: a systematic literature review. Clin Exp Rheumatol 2012;30(4 Suppl. 73):S145-8.

[3] Robinson PC, Merriman TR, Herbison P, Highton J. Hospital admissions associated with gout and their comorbidities in New Zealand and England 1999-2009. Rheumatology (Oxford) 2013;52:118-26.

[4] Lee G, Roberts L. Healthcare burden of in-hospital gout. Intern Med J 2012 Nov; 42(11):1261-3.

[5] Kim KY, Schumacher HR, Hunsche E, Wertheimer AI, Kong SH. A literature review of the epidemiology and treatment of acute gout. Clin Ther 2003;25: 1593-617.

[6] Nobili A, Licata G, Salerno F, Pasina L, Tettamanti M, Franchi C, et al. Polypharmacy, length of hospital stay, and in-hospital mortality among elderly patients in internal medicine wards. The REPOSI study, Eur I Clin Pharmacol 2011;67:507-19.

[7] Franchi C, Nobili A, Mari D, Tettamanti M, Djade CD, Pasina L, et al, on behalf of REPOSI Investigators. Risk factors for hospital readmission of elderly patients. Eur J Intern Med 2013 Jan;24(1):45-51. http://dx.doi.org/10.1016/j.ejim.2012.10.005 [Epub 2012 Nov 8]

[8] Parmelee PA, Thuras PD, Katz IR, Lawton MP. Validation of a measure of physical burden at autopsy: the cumulative illness rating scale. J Am Geriatr Soc 1995;43: 130-7. 
[9] Shah S, Vanclay F, Cooper B. Improving the sensitivity of the Barthel Index for stroke rehabilitation. J Clin Epidemiol 1989;42:703-9.

[10] Katzman R, Brown T, Fuld P, Peck A, Schechter R, Schimmel H. Validation of a short orientation-memory-concentration test of cognitive impairment. Am J Psychiatry 1983;140:734-9.

[11] Hickie C, Snowdon J. Depression scales for the elderly: GDS, Gilleard, Zung. Clin Gerontol 1987;6:51-3.

[12] Salaffi F, De Angelis R, Grassi W, MArche Pain Prevalence, INvestigation Group (MAPPING) study. Prevalence of musculoskeletal conditions in an Italian population sample: results of a regional community-based study. I. The MAPPING study. Clin Exp Rheumatol 2005;23:819-28.

[13] Smith EU, Díaz-Torné C, Perez-Ruiz F, March LM. Epidemiology of gout: an update. Best Pract Res Clin Rheumatol 2010;24:811-27.
[14] Krishnan E, Svendsen K, Neaton JD, Grandits G, Kuller LH. Long-term cardiovascular mortality among middle-aged men with gout. Arch Intern Med 2008; $168: 1104-10$.

[15] Krishnan E, Baker JF, Furst DE, Schumacher HR. Gout and the risk of acute myocardial infarction. Arthritis Rheum 2006;54:2688-96.

[16] Riedel AA, Nelson M, Wallace K, Joseph-Ridge N, Cleary M, Fam AG. Prevalence of comorbid conditions and prescription medication use among patients with gout and hyperuricemia in a managed care setting. J Clin Rheumatol 2004;10:308-14.

[17] Annemans L, Spaepen E, Gaskin M, et al. Gout in the UK and Germany: prevalence, comorbidities and management in general practice 2000-2005. Ann Rheum Dis 2008;67:960-6.

[18] Bhole V1, Choi JW, Kim SW, de Vera M, Choi H. Serum uric acid levels and the risk of type 2 diabetes: a prospective study. Am J Med Oct 2010;123(10):957-61. 